\title{
HIV-1 infection and perinatal mortality in Zimbabwe
}

\author{
C G A Aiken
}

\begin{abstract}
As part of a survey of the causes of perinatal mortality at Mpilo Maternity Hospital, 220 neonatal deaths and the mothers of 221 stillbirths were tested for HIV-1 antibodies. The HIV positive rate in neonatal deaths was $23.6 \%$ (95\% confidence interval (CI) 18.0 to $29.2 \%)$, significantly higher than $15.4 \%(95 \%$ CI 10.6 to $20.1 \%$ ) in stillbirths. Perinatal deaths from congenital malformations, birth asphyxia, pregnancy induced hypertension, placental abruption, and other non-infectious causes had similar low HIV positive rates averaging $8 \cdot 1 \%$ (95\% CI 3.9 to $12 \cdot 3 \%)$. Deaths from septicaemia had a significantly greater rate of $39.3 \%(95 \% \mathrm{CI} 27.0$ to $51.6 \%)$ and the highest rate of $72 \cdot 2 \%(95 \%$ CI 51.5 to $92.9 \%)$ was found in deaths from congenital infection other than syphilis, indicating that maternal HIV infection predisposes to neonatal septicaemia and congenital infection. Unexplained stillbirths also had a significantly greater rate of $22.4 \%(95 \%$ CI 10.7 to $34 \cdot 1 \%)$, presumably because some died from unrecognised infection. The rate in deaths from congenital syphilis was $17 \cdot 4 \%$ (95\% CI 9.6 to $25 \cdot 2 \%$ ), indicating a significant but weak association between these two sexually transmitted diseases in Bulawayo. The rate in deaths from hyaline membrane disease was not significantly greater at $15 \cdot 0 \%$ (95\% CI 6.0 to $24 \cdot 0 \%$ ). By predisposing to infection, maternal HIV infection was estimated to increase the stillbirth rate by 1.6 times and the neonatal mortality rate by 2.7 times. It predisposed equally to early and late onset neonatal septicaemia, but more to infection from streptococci and staphylococci than from Gram negative enterobacteria. HIV positive deaths from congenital infection had respiratory distress and usually intrauterine growth retardation, hepatosplenomegaly, and congenital pneumonia on lung histology.
\end{abstract}

In Zimbabwe and other Central African countries, the epidemic of heterosexually transmitted HIV-1 infection is now a major health problem. Studies have estimated that between $7 \%$ and $39 \%$ of HIV positive mothers transmit the infection to their babies. ${ }^{1-8}$ The lowest transmission rate was found in Europe among asymptomatic intravenous drug abusers who rarely breast fed, ${ }^{1}$ whereas the highest rate was found in two studies in Central Africa among breast feeding mothers, one sixth of whom had symptoms of HIV disease. ${ }^{78}$ Most infected babies become ill in the first year but rarely in the neonatal period. ${ }^{2} 910$ In Europe and America
HIV positive mothers had stillbirth and neonatal mortality rates of about $10 / 1000$ births, similar to those in control HIV negative mothers, ${ }^{2}{ }^{10-12}$ but had higher infant mortality rates of around 20/1000 births. $^{2} 4$ In contrast HIV positive mothers in Central Africa had stillbirth rates of about $20 / 1000$ births, similar to those in control mothers, ${ }^{7813}$ but had higher neonatal mortality rates of $60 / 1000$ births, compared with $15 / 1000$ in controls, ${ }^{73}$ and a much higher infant mortality rate of $150 / 1000$ births, compared with $25 / 1000$ in controls. ${ }^{7}$ This indicates that infected babies die earlier in Africa and suggests that, in addition to being a major cause of infant mortality, ${ }^{7}$ HIV infection may be a significant cause of neonatal mortality, at least in urban communities with a high prevalence of infection.

A survey of the causes of perinatal mortality in Bulawayo, Zimbabwe, found that congenital syphilis, birth asphyxia, hyaline membrane disease, neonatal septicaemia, congenital malformations, pregnancy induced hypertension, and placental abruption together accounted for $80 \%$ of the deaths. ${ }^{14}$ HIV infection predisposes infants to septicaemia, ${ }^{15}{ }^{16}$ and it was therefore postulated to be a factor contributing to the high rate of neonatal septicaemia in Bulawayo. One study in Africa found that the proportion of HIV positive mothers that delivered prematurely was greater than in controls, ${ }^{17}$ and another study showed that prematurity was associated with HIV seropositivity, ${ }^{17}$ suggesting that infection might predispose to hyaline membrane disease by causing prematurity. In five studies, three from Africa ${ }^{8} 1318$ and two from Europe ${ }^{11}$ and America, ${ }^{13}$ however, no increase in prematurity compared with controls was found. It is now established that HIV infection does not cause congenital malformations, 24571819 and there is little or no evidence that it is associated with birth asphyxia, ${ }^{7}$ pregnancy induced hypertension, ${ }^{12}$ or placental abruption. However, being a sexually transmitted disease, it may be associated with syphilis.

In order to determine what if any contribution maternal HIV infection makes to the high perinatal mortality rate in Bulawayo, the HIV status of consecutive neonatal deaths and stillbirths was tested and from this the proportion of deaths from each cause that were HIV positive was calculated. A high proportion would suggest that HIV infection contributes to the cause of death, whereas a low proportion would indicate no contribution.

Patients and methods

Over a seven month period, as part of a survey 
of the causes of perinatal mortality at Mpilo Maternity Hospital in Bulawayo, ${ }^{14}$ blood taken for syphilis serology from all neonatal deaths and from the mothers of all stillbirths was anonymously tested for HIV-1 antibodies using two enzyme immunoassay tests (Abbott Recombinant HIV-1 EIA and either Vironostika antiHTLV-III MicroELISA or Du Pont HIV-1 ELISA). If the two tests gave discrepant results, both tests were repeated until concordant results were obtained. As the tests detect IgG antibodies that cross the placenta, a positive result in the infant or mother was taken to indicate maternal HIV infection. The results were kept confidentially and were not made available to the mothers or staff of the hospital. In order to prevent a bias in determing the cause of death, the results were not matched to the patients until the end of the survey when the causes of death had already been established, as described previously. ${ }^{14}$ The results were analysed by calculating the proportion, with $95 \%$ confidence intervals $(\mathrm{CI})$, of deaths from each cause that were HIV positive. These proportions were then compared using $\chi^{2}$ tests.

\section{Results}

Over the seven month period, HIV serology results were obtained in 220 out of a total of 235 (94\%) neonatal deaths and in 221 out of a total of $271(82 \%)$ stillbirths. Forty two of the 50 stillbirths with no results were delivered in the first and last months of the survey, and in the intervening five months results were obtained in $96 \%$ of stillbirths. Seven of the neonatal deaths with results were in fact babies who died after 28 days, but were included because they died before discharge from the neonatal unit. The proportions of perinatal deaths attributed to each cause were not significantly different in the sample with HIV results compared with the whole group of deaths analysed over the 12 month survey period $(p>0 \cdot 10<0 \cdot 90) . .^{14}$

Out of the total of 441 perinatal deaths tested, 86 were HIV positive and 355 were HIV negative, indicating that $19 \cdot 5 \%(95 \% \mathrm{CI} 15 \cdot 8$ to $23 \cdot 2 \%$ ) of perinatal deaths were HIV positive. The proportion of neonatal deaths that were HIV positive was $23.6 \%(95 \%$ CI 18.0 to
$29 \cdot 2 \%$ ), significantly greater than the proportion of $15 \cdot 4 \%$ (95\% CI $10 \cdot 6$ to $20 \cdot 1 \%)$ found in stillbirths $(p<0.05)$, suggesting that maternal HIV infection is more likely to contribute to neonatal deaths than to stillbirths. The results from the stillbirths and neonatal deaths were combined and analysed according to the mother's age (figure) and the cause of death (table 1).

As shown in the figure, the proportion of mothers that were HIV positive was significantly greater in the 20-24 year group than in either the $25-29$ or the $30-34$ year group, suggesting that women under 25 years are most at risk of acquiring HIV infection.

The proportions of perinatal deaths that were HIV positive are shown for each cause of death in table 1. Deaths from congenital malformations, birth asphyxia, pregnancy induced hypertension, placental abruption, and other non-infectious causes excluding hyaline membrane disease all had similar low proportions, with a combined HIV positive rate of $8 \cdot 1 \%$ $(95 \%$ CI 3.9 to $12 \cdot 3 \%)$. This rate was used as a baseline against which to compare the rates in deaths from the remaining causes. The rate in

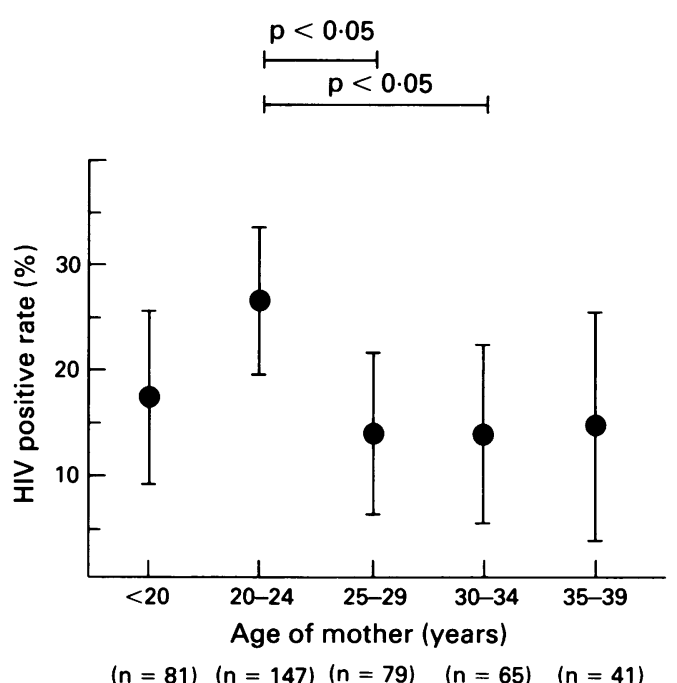

Relation between mothers' age and HIV positive rate. Bars represent $95 \%$ CI of the rate. Fourteen mothers over 39 years were tested and three were HIV positive.

Table 1 The HIV positive rate for each cause of perinatal death

\begin{tabular}{|c|c|c|c|c|c|c|c|c|c|c|}
\hline & \multicolumn{2}{|c|}{ Stillbirths } & \multicolumn{2}{|c|}{ Neonatal deaths } & \multicolumn{2}{|c|}{ Perinatal deaths } & \multirow{2}{*}{$\begin{array}{l}\text { HIV+ } \\
\text { rate } \\
(\%)\end{array}$} & \multirow[t]{2}{*}{$95 \% C I$} & \multirow[t]{2}{*}{$x^{2}$} & \multirow[t]{2}{*}{ p Value } \\
\hline & $H I V-$ & $H I V+$ & $H I V-$ & $H I V+$ & $H I V-$ & $H I V+$ & & & & \\
\hline $\begin{array}{l}\text { Congenital malformations } \\
\text { Birth asphyxia } \\
\text { Pregnancy induced hypertension } \\
\text { Placental abruption } \\
\text { Other non-infectious causes* } \\
\text { Hyaline membrane disease } \\
\text { Congenital syphilis } \\
\text { Unexplained stillbirths } \\
\text { Neonatal septicaemia† } \\
\text { Congenital infection }\end{array}$ & $\begin{array}{r}10 \\
38 \\
21 \\
17 \\
9 \\
0 \\
48 \\
38 \\
6 \\
0\end{array}$ & $\begin{array}{r}2 \\
5 \\
2 \\
1 \\
0 \\
0 \\
8 \\
11 \\
4 \\
1\end{array}$ & $\begin{array}{r}16 \\
24 \\
0 \\
0 \\
13 \\
51 \\
28 \\
0 \\
31 \\
5\end{array}$ & $\begin{array}{r}1 \\
1 \\
0 \\
0 \\
1 \\
9 \\
8 \\
0 \\
20 \\
12\end{array}$ & $\begin{array}{r}26 \\
62 \\
21 \\
17 \\
22 \\
51 \\
76 \\
38 \\
37 \\
5\end{array}$ & $\begin{array}{r}3 \\
6 \\
2 \\
1 \\
1 \\
9 \\
16 \\
11 \\
24 \\
13\end{array}$ & $\begin{array}{l}15 \cdot 0 \\
17 \cdot 4 \\
22 \cdot 4 \\
39 \cdot 3 \\
72 \cdot 2\end{array}$ & $\begin{array}{r}6.0 \text { to } 24 \cdot 0 \\
9.6 \text { to } 25 \cdot 2 \\
10 \cdot 7 \text { to } 34 \cdot 1 \\
27 \cdot 0 \text { to } 51 \cdot 6 \\
51.5 \text { to } 92.9\end{array}$ & $\begin{array}{r}2 \cdot 34 \\
5 \cdot 01 \\
7.67 \\
31 \cdot 14 \\
53 \cdot 66\end{array}$ & $\begin{array}{l}>0.1 \\
<0.05 \\
<0.01 \\
<0.001 \\
<0.001\end{array}$ \\
\hline Total & 187 & 34 & 168 & 52 & 355 & 86 & & & & \\
\hline
\end{tabular}

The HIV positive rate for each of the last five causes was compared using $\chi^{2}$ tests with the average rate for the first five causes.

${ }^{*}$ Other non-infectious causes of stillbirths were postmaturity $(n=4)$, diabetes $(n=3)$, placenta praevia $(n=1)$, and cord $k$ not $(n=1)$ and of neonatal deaths were meconium aspiration syndrome $(n=7)$, birth trauma $(n=3)$, kernicterus $(n=2)$, milk aspiration $(n=1)$, and posthaemorrhagic hydrocephalus $(n=1)$.

†Ten fresh stillbirths who died after prolonged rupture of membranes or maternal fever with features of amnionitis were presumed to have had septicaemia and were therefore included in neonatal septicaemia. In only one was a blood culture obtained and it grew Escherichia coli. Six of the neonatal deaths from septicaemia had typical features of septicaemia but negative blood cultures. The HIV positive rate in the 46 blood culture positive deaths from septicaemia was $39 \cdot 1 \%$ ( $95 \%$ CI $25 \cdot 0$ to $53 \cdot 2 \%$, very similar to the rate in the group as a whole. 
hyaline membrane disease was not significantly greater at $15.0 \%(95 \%$ CI 6.0 to $24.0 \%)$ whereas the rate in congenital syphilis was significantly greater but was only $17 \cdot 4 \%$ (95\% CI 9.6 to $25 \cdot 2 \%)$. Unexplained stillbirths also had a significantly greater rate of $22 \cdot 4 \%$ (95\% CI $10 \cdot 7$ to $34 \cdot 1 \%)$. However, the rate in neonatal septicaemia was much greater at $39 \cdot 3 \%$ (95\% CI $27 \cdot 0$ to $51 \cdot 6 \%$ ) and the highest rate of $72 \cdot 2 \%$ (95\% CI 51.5 to $92.9 \%)$ was found in deaths caused by congenital infections other than syphilis and early onset septicaemia. Details regarding these 18 deaths have been reported separately. ${ }^{14}$ Of the 13 that were HIV positive, three died after 28 days from AIDS with failure to thrive and multiple episodes of septicaemia, nine died in the first 14 days with features suggesting congenital infection, and one was a fresh small for gestational age stillbirth with hepatosplenomagaly. Of the five who were HIV negative, one had congenital tuberculosis with miliary foci of caseous necrosis in the lung and liver containing acid fast bacilli and few inflammatory cells, one probably had herpes simplex infection with a vesicular skin rash, microabscesses in the lung, and massive hepatic necrosis, and three had non-specific features of congenital infection.

After completion of this study all neonatal deaths from septicaemia and congenital infection were HIV tested and the parents counselled. Over the next seven months, 47 out of 51 neonatal deaths from septicaemia were tested and 22 were HIV positive giving a rate of $46.8 \%$ (95\% CI 32.5 to $61 \cdot 1 \%)$. These deaths were combined with those from the previous seven month study period in order to determine if there were any differences between HIV positive and HIV negative cases in birth weight, age at death, or bacteria cultured. As shown in table 2 , no significant differences in birth weight or age at death were found, but there were significant differences in the bacteria responsible. The HIV positive rate in deaths from streptococcal and staphylococcal septicaemia was $52 \cdot 5 \%(95 \%$ CI 37.0 to $68 \cdot 0 \%)$, significantly higher than the rate of $23.8 \%(95 \%$ CI 10.9 to $36.7 \%$ ) found in deaths from Gram negative enterobacteria $(p<0.01)$. In three HIV positive cases, haemophilus, bacillus, and acinetobacter species were isolated. In the latter two cases the same organism was grown in two separate blood culture samples, and in eight HIV positive cases with typical features of septicaemia no bacteria were grown. Nine out of 11 babies who died from pulmonary or gastrointestinal haemorrhage caused by septicaemia were HIV positive.

During the seven months after this study all neonatal deaths with features of congenital infection were HIV positive. Over the whole 14 month period, all eight surviving infants discharged from the neonatal unit with features of congenital infection were HIV tested and three were HIV positive. Table 3 gives a summary of the clinical, laboratory, and histological features of all the HIV positive and HIV negative infants with congenital infection excluding the five cases of AIDS, congenital tuberculosis, and probable herpes simplex infection described above. Most infants in both groups were small for gestational age with respiratory distress, hepatosplenomegaly and less commonly petechiae and if they survived after 48 hours developed conjugated hyperbilirubinaemia, anaemia, and thrombocytopenia. There were five infants with microcephaly and other features of congenital infection, all of whom survived to discharge. Two of these were HIV positive and both had high rubella IgG titres which increased further on repeat testing. Two of the three HIV negative cases had rubella IgG titres of 1:64 or greater but repeat titres were not obtained. Six surviving infants with microcephaly but no other features of congenital infection were HIV tested and all were negative. Rubella IgG titres obtained in four of them were 1:64 or greater in all cases and one on repeat testing had an increased titre. Serological tests for cytomegalovirus, toxoplasmosis, and herpes infections were incomplete and produced no diagnostically useful results.

In order to determine how much maternal
Table 2 Comparison of HIV positive and HIV negative deaths from septicaemia

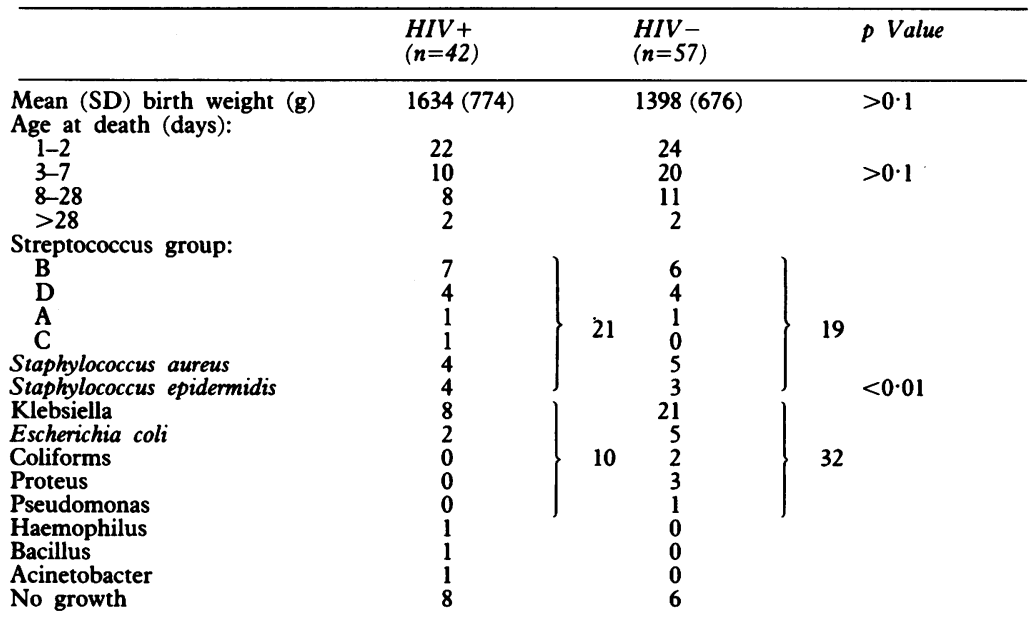

This table excludes the nine stillbirths included in table 1 who were presumed to have died from septicaemia but had no blood culture samples obtained.
Table 3 Comparison of HIV positive and HIV negative infants with congenital infection

\begin{tabular}{|c|c|c|}
\hline & $H I V+$ & $H I V-$ \\
\hline $\begin{array}{l}\text { No (No who died) } \\
\text { Mean (SD) birth weight (g) } \\
\text { Age at death (days): }\end{array}$ & $\begin{array}{c}18(15) \\
1779(759)\end{array}$ & $\begin{array}{c}8(3) \\
2162(629)\end{array}$ \\
\hline Age at death (days): & 11 & 1 \\
\hline $3-7$ & 2 & 1 \\
\hline $8-28$ & 2 & 0 \\
\hline$>28$ & 0 & 1 \\
\hline \multicolumn{3}{|l|}{ Features: } \\
\hline Small for gestational age & $13 / 18$ & $6 / 8$ \\
\hline Respiratory distress & $15 / 17$ & $4 / 8$ \\
\hline Hepatomegaly & $11 / 18$ & $7 / 8$ \\
\hline Splenomegaly & $9 / 18$ & $8 / 8$ \\
\hline Petechia & $5 / 18$ & $4 / 8$ \\
\hline Convulsions & $3 / 17$ & $1 / 8$ \\
\hline Microcephaly & $2 / 18$ & $3 / 8$ \\
\hline Microphthalmia & $1 / 18$ & $0 / 8$ \\
\hline Hydrops & $0 / 18$ & $1 / 8$ \\
\hline Anaemia & $5 / 8$ & $3 / 7$ \\
\hline Thrombocytopenia & $5 / 7$ & $3 / 6$ \\
\hline Conjugated hyperbilirubinaemia & $4 / 7$ & $4 / 8$ \\
\hline Septicaemia & $2 / 17$ & $1 / 8$ \\
\hline \multicolumn{3}{|l|}{$\begin{array}{l}\text { Histology: } \\
\text { Hial }\end{array}$} \\
\hline Generalised atelectasis & & $1 / 3$ \\
\hline Congenital pneumonia & $5 / 9$ & $1 / 3$ \\
\hline Meconium aspiration & $1 / 9$ & $0 / 3$ \\
\hline Hepatitis & $1 / 9$ & $2 / 3$ \\
\hline
\end{tabular}


HIV infection increases perinatal mortality, the prevalence of HIV infection in mothers delivering at Mpilo Maternity Hospital was first estimated. In mothers without syphilis this rate should be similar to the $8 \cdot 1 \%$ found in perinatal deaths not associated with HIV infection, that is congenital malformations, birth asphyxia, pregnancy induced hypertension, placental abruption, and other non-infectious causes excluding hyaline membrane disease, which may be associated with HIV infection. In mothers with syphilis this rate should be similar to the $17 \cdot 4 \%$ found in perinatal deaths from congenital syphilis. During the survey $11 \cdot 0 \%$ of mothers attending antenatal clinics in Bulawayo had positive syphilis serology and using this figure the prevalence of HIV infection among mothers delivering at Mpilo Maternity Hospital was estimated to be $9 \cdot 1 \%$. This rate should be less than the rate in mothers resident in Bulawayo because some of the deliveries at the hospital are referred from rural areas with a lower prevalence of HIV infection. Using this estimate and the HIV positive rates for each cause of death given in table 1 , the number of HIV positive and negative deliveries, stillbirths, and neonatal deaths were calculated over the whole 12 month survey period. ${ }^{14}$ From these figures, HIV positive mothers were found to have a perinatal mortality rate $2 \cdot 1$ times greater, a stillbirth rate 1.6 times greater, and a neonatal mortality rate $2 \cdot 7$ times greater than HIV negative mothers.

\section{Discussion}

This study demonstrates that maternal HIV infection contributes to perinatal mortality in Zimbabwe by predisposing to neonatal septicaemia and congenital infection. There is no evidence that HIV infection increases the risk of congenital malformations, birth asphyxia, pregnancy induced hypertension, placental abruption, and other non-infectious causes excluding hyaline membrane disease. ${ }^{2} 457121819$ The HIV positive rate in deaths from these causes was $8 \%$, reflecting the prevalence of HIV infection in mothers without syphilis delivering at Mpilo Maternity Hospital. This rate is similar to the rates of $6 \%$ and $12 \%$ found in 1987 in mothers in Kinshasa, Zaire ${ }^{7}$ and Lusaka, $\mathrm{Zambia}^{8}$ respectively. The HIV positive rate in deaths from neonatal septicaemia and congenital infection was significantly greater at $39 \%$ and $72 \%$ respectively, indicating that maternal HIV infection predisposes to deaths from these conditions. The rate in unexplained stillbirths was also significantly greater at $22 \%$ presumably because some of these stillbirths died from unrecognised infection. The rate in deaths from congenital syphilis was significantly increased at $17 \%$, indicating a weak association between these two heterosexually transmitted diseases in Bulawayo. In Nairobi no association was found. ${ }^{19}$ Unlike HIV infection, untreated early syphilis greatly increases perinatal mortality and is the leading cause of perinatal death in Bulawayo, but could be effectively controlled by screening all mothers at about 20 weeks and treating those with positive syphilis serology. ${ }^{14}$
The rate in deaths from hyaline membrane disease was not significantly increased at $15 \%$, although perhaps a larger study would show a significant association if HIV infection predisposes to preterm delivery. ${ }^{7} 17$

Deaths from septicaemia and congenital infection occur mainly after birth and HIV positive mothers were estimated to have a neonatal mortality rate of $2 \cdot 7$ times greater and a stillbirth rate 1.6 times greater than HIV negative mothers. In Kinshasa HIV positive mothers had more than four times the neonatal mortality rate but a similar stillbirth rate to HIV negative controls. ${ }^{7}$ Most infected infants born to HIV positive mothers do not become sick until after the neonatal period, ${ }^{18}$ however, and the major impact of HIV infection is to increase infant mortality rather than perinatal mortality. In Kinshasa HIV positive mothers had more than six times the infant mortality rate of HIV negative controls. ${ }^{7}$ Unlike syphilis, HIV infection is far more difficult to control. In Bulawayo, like other parts of Central Africa, ${ }^{18}$ young women under the age of 25 years are most at risk and for health education to have any effect it should be directed at adolescents at school.

Maternal HIV infection could predispose to neonatal septicaemia by either reducing passively acquired immunity from the mother without infecting the baby or reducing the baby's own immunity by direct infection. It predisposes equally to early onset septicaemia acquired from the mother and to late onset hospital acquired septicaemia, but predisposes more to septicaemia caused by Gram positive cocci than to septicaemia caused by Gram negative enterobacteria. These observations suggest that a reduction in passively acquired immunity especially to streptococcal and staphylococcal infection is important. HIV infection may predispose to neonatal septicaemia simply by causing preterm delivery, but this is unlikely to be important because the mean birth weight of HIV positive and HIV negative deaths from septicaemia were similar and significantly greater than the mean birth weight of deaths from hyaline membrane disease $(p<0.001)$, who had a significantly lower HIV positive rate $(\mathrm{p}<0.01)$. In Kinshasa chorioamnionitis was common in mothers with AIDS but was rare in asymptomatic HIV positive mothers. ${ }^{7}$

Maternal HIV infection could predispose to congenital infection by reducing maternal immunity to rubella, cytomegalovirus, toxoplasmosis, and other pathogens allowing them to infect the fetus more easily, or the HIV virus could infect the fetus and be responsible on its own for the features of congenital infection. Most of the features found in HIV positive cases were similar to those in recognised congenital infections including intrauterine growth retardation, hepatosplenomegaly, conjugated hyperbilirubinaemia, anaemia, and thrombocytopenia. However respiratory distress was a prominent feature and major cause of death with lung histology in five out of nine cases showing congenital pneumonia with intralveolar haemorrhage. In this context it is interesting to note than nine out of the 11 babies who died from pulmonary or gastrointestinal haemorrhage 
caused by neonatal septicaema were HIV positive. Microcephaly was an unusual feature seen in only two surviving HIV positive cases both of whom were probably infected with rubella.

In order to determine how maternal HIV infection predisposes to septicaemia and congenital infection more sophisticated investigations are needed but are not at present available in Zimbabwe. The polymerase chain reaction could be used to determine what proportion of the deaths are actually infected with the HIV virus, ${ }^{20}$ viral culture being more difficult and antigen tests being negative at birth even in infected babies. ${ }^{11}$ Total IgM measurements soon after birth could help establish the diagnosis of congenital infection and tests for rubella or cytomegalovirus specific IgM could be used to confirm infection by these viruses.

I am most grateful to Dr N T Chaibva, the medical superintendent of Mpilo Central Hospital, for permission to perform this study, to all the staff of the Maternity Hospital microbiology and histopathology departments for their help, and especially to Sr A M Luza, Sr F M Moyo, Dr J J Valdes Gomez, Dr F J Gonzales de Chaves, Dr Z Sibanda, Dr M K Khan, Dr R M A W El-Gammal, Dr R A Dlodlo, and Mrs H E Aiken for their invaluable assistance.

1 Mok JYQ, Hague RA, Yap PL, et al. Vertical transmission of HIV: a prospective study. Arch Dis Child 1989;64:1140-5.

2 European Collaborative Study. Children born to women with HIV-1 infection: natural history and risk of transmission. Lancet 1991;337:253-60.

3 Andiman WA, Simpson J, Olson B, Dember L, Silva TJ, Miller G. Rate of transmission of human immunodeficiency virus type 1 infection from mother to child and short-term outcome of neonatal infection. Am $\mathcal{J}$ Dis Child 1990;144: 758-66.

4 Blanche S, Rouzioux C, Guihard Moscato M-L, et al. A prospective study of infants born to women seropositive for human immuno-deficiency virus type $1 . N \mathrm{Engl} f \mathrm{Med}$ 1989;320:1643-8.
5 Italian Multicentre Study. Epidemiology, clinical features, and prognostic factors of paediatric HIV infection. Lancet 1988;ii:1043-5.

6 Goedert JJ, Mendez H, Drummond JE, et al. Mother-toinfant transmission of human immunodeficiency virus type 1989;ii: $1351-4$.

7 Ryder RW, Nsa W, Hassig SE, et al. Perinatal transmission of the human immunodeficiency virus type 1 to infants of seropositive women in Zaire. $N$ Engl f Med 1989;320: $1637-42$.

8 Hira SK, Kamanga J, Bhat GJ, et al. Perinatal transmission of HIV-1 in Zambia. BMF 1989;299:1250-2.

9 Scott GB, Hutto C, Makuch RW, et al. Survival in children with perinatally acquired human immunodeficiency virus type 1 infection. N Engl f Med 1989;321:1791-6.

10 Gloeb DJ, O'Sullivan MJ, Efantis J. Human immunodeficiency virus infection in women. 1 . The effects of human immunodeficiency virus on pregnancy. Am $\mathcal{F} O$ bstet Gynecol 1988;159:756-61.

11 Johnstone FD, MacCallum L, Brettle R, Inglis JM, Peutherer JF. Does infection with HIV affect the outcome of pregnancy? $B M F$ 1988;296:467.

12 Selwyn PA, Schoenbaum EE, Davenny K, et al. Prospective study of human immunodeficiency virus infection and pregnancy outcomes in intravenous drug abusers. $\neg A M A$ pregnancy outcom

13 Lallemont M, Lallemont-Le-Coeur S, Cheynier D, et al. Mother-child transmission of HIV-1 and infant survival in Brazzaville, Congo. AIDS 1989;3:643-6.

14 Aiken CGA. The causes of perinatal mortality in Bulawayo, Zimbabwe. Cent Afr J Med 1992 (in press).

15 Lepage P, Van de Perre P, Nsengumuremyi F, Van Goethem C, Bogaerts J, Hitimana DG. Bacteraemia as predictor of HIV infection in African children. Acta Paediatr Scand 1989;78:763-6.

16 Howard-Gardiner H, Roberts PD, Dunn PM. Congenital human immunodeficiency virus (HIV) infection in the Bahamas. Br F Obstet Gynaecol 1989;96:140-3.

17 Temmerman M, Plummer FA, Mizra NB, et al. Infection with HIV as a risk factor for adverse obstetrical outcome. AIDS 1990;4:1087-93.

18 Lepage P, Dabis F Hitimana D-G, et al. Perinatal transmission of HIV-1: lack of impact of maternal HIV infection on characteristics of livebirths and on neonatal mortality in on characteristics of livebirths and on ne
Kigali, Rwanda. AIDS 1991;5:295-300.

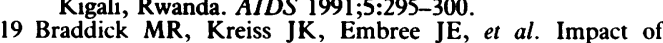
maternal HIV infection on obstetrical and early neonatal outcome. AIDS 1990;4:1001-5.

20 Rogers MF, Ou C-Y, Rayfield M, et al. Use of the polymerase chain reaction for early detection of the proviral sequence of human immunodeficiency virus in infants born to seropositive mothers. $N$ Engl f Med 1989;320:1649-54. 21 Borkowsky W, Kransinski K, Paul D, et al. Human Pediatr 1989;114:940-5. 